\title{
Morphology of Alveolar Macrophage Uptake of Nano-Carbon Materials in Rat Lung
}

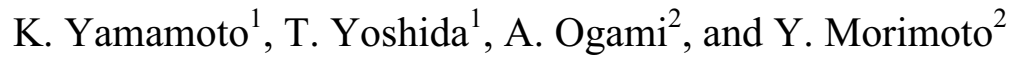 \\ 1. National Institute of Advanced Industrial Science and Technology (AIST), Tsukuba, Japan \\ 2. University of Occupational and Environmental Health, Kitakyushu, Japan
}

Nano-carbon materials such as fullerenes and carbon nanotubes were attractive materials for industrial applications in many fields. However, the toxicity of these nano-carbon materials for the human was not clear, so the toxicity and risk assessment of nano-carbon materials were important. In this study, in vivo toxicity tests of fullerene, multi-wall carbon nanotube (MWCNT), and single-wall carbon nanotube (SWCNT) were performed using the intratracheal instillation in rat lung, and the morphology of alveolar macrophage in rat lung was examined by using transmission electron microscope (TEM).

The test material solutions of fullerenes, MWCNTs, and SWCNTs were intratracheally instilled in the rat lung. Tween 80 dispersant of $0.1 \mathrm{~g} / 1$ was used for fullerenes solution and Triton-X dispersant of $1 \mathrm{~g} / 1$ content was used for MWCNT and SWCNT solutions. The diameter of fullerene particles was $20 \mathrm{~nm}$. The instilled mount of fullerenes was $0.1,0.2$, or $1.0 \mathrm{mg}$ for each body. The average diameter of MWCNT was $48 \mathrm{~nm}$, and the length was ranging from 0.2 to $6 \mu \mathrm{m}$. The instilled mounts of MWCNT were 0.2 or $1.0 \mathrm{mg}$ for each body. The average diameter of SWCNT was $1.8 \mathrm{~nm}$, and SWCNTs were bundled. The length of SWCNT bundles was ranging from 0.2 to $4 \mu \mathrm{m}$. The average width of bundles was $44 \mathrm{~nm}$. The instilled mount of SWCNT was 0.2 or $0.4 \mathrm{mg}$ for each body. NiO nano-particles with average diameter of $26 \mathrm{~nm}$ were used as positive control materials. The instilled mount of $\mathrm{NiO}$ was $0.2 \mathrm{mg}$ for each body.

The lung tissues after three days, one week, one month, three months and six months from the instillation were observed by TEM. The lung tissue was fixed using glutaraldehyde and osmium tetroxide solution, and then dehydrated in ethanol, and embedded in epoxy resin. Ultrathin sections were cut on a diamond knife with microtomy. The stained specimens were also examined using conventional biological TEM (Hitachi, H-7600). The zero loss imaging of the non-stained tissue was performed by an energy-filtering TEM with the high-resolution objective lens (Zeiss, EM922HR).

For fullerenes, the black particles are observed in the phagosome of the alveolar macrophages after a week instillation exposure of $0.2 \mathrm{mg}$ dose (Figure 1(a)). According to the electron diffraction analysis, these particles are identified as the fullerenes. Fullerenes particles keep the fcc crystalline structure. As the diameter of fullerenes is $20 \mathrm{~nm}$, fullerenes keep the particle size. The most of fullerenes particles are observed in the alveolar macrophages and a part of fullerenes are in the alveolar cells. Fullerenes still remain in the alveolar macrophages or the alveolar cells after 3 months instillation exposure of $0.2 \mathrm{mg}$ dose (Figure 1(b)), however the inflammation of the alveolar cells is not observed.

Figure 2 (a) shows alveolar macrophage in lung after 3 days instillation exposure of $0.2 \mathrm{mg}$ dose of MWCNTs. Fibrous objects are observed at the cytoplasm of macrophage. According to high- resolution analysis, these fibers are MWCNTs, and MWCNTs keep the tube and the graphitic structures. This is because that graphite is chemically stable. In the tissue after 3 months instillation exposure of $0.2 \mathrm{mg}$ dose, the inflammation of the alveolar cells is observed and accumulation of alveolar macrophages is 
also observed in the inflammation (Figure 2(b)). This accumulation shows that clearance of MWCNT is slower than fullerenes.

TEM images of the alveolar cells after 3 days instillation exposure of SWCNTs solution of $0.2 \mathrm{mg}$ dose are shown in Figure 3 (a)-(b). The black fibrous objects are observed in the phagosome of the alveolar macrophages. Accumulation of alveolar macrophages and the inflammations are observed (Figure 3 (b)). According to high-resolution observations, these fibrous objects are SWCNTs. SWCNTs bundles are taken up by macrophage. In the tissue after 3 months instillation exposure of $0.2 \mathrm{mg}$ dose, the foam cell formation in alveolar macrophage is observed (Figure 3 (c)). The persistent inflammation and the foam cell formation in the alveolar macrophage indicate that SWCNTs have the toxic potentials.

We summarized that the toxic potential of nano-carbon materials in the same dose of $0.2 \mathrm{mg}$ is greater in the order of SWCNT, MWCNT, and fullerenes, because of the foam cell forming in alveolar macrophage and the persistent inflammation.
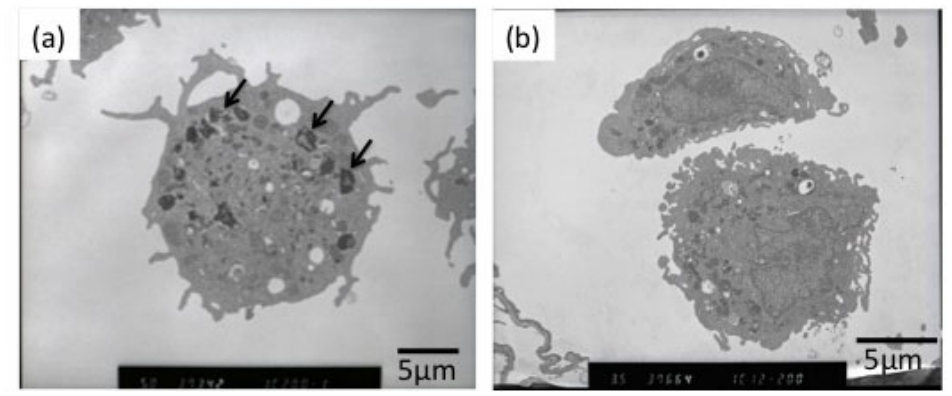

Figure 1. Alveolar macrophage in the rat lung after 1 week (a), and 3 months (b) instillation of fullerenes.
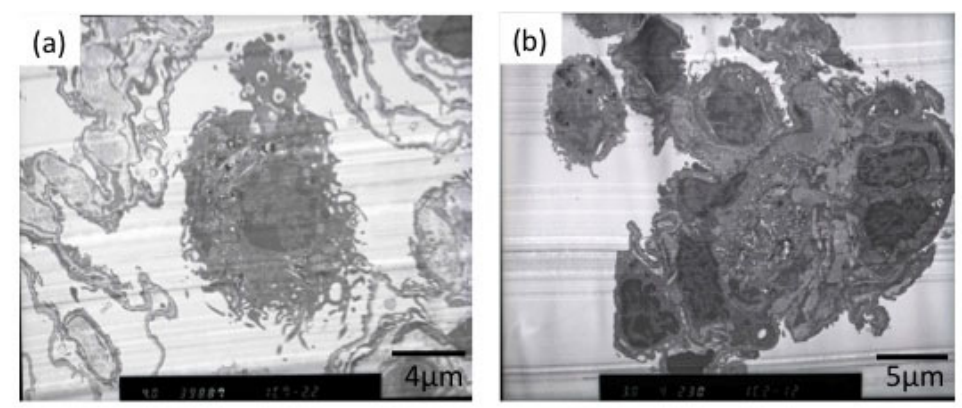

Figure 2. Alveolar macrophage in the rat lung after 1 week (a), and 3 months (b) instillation of MWCNTs.

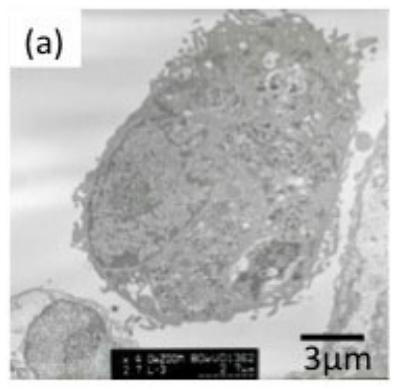

\section{(b)}

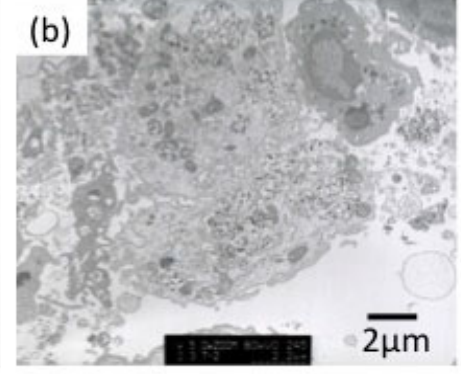

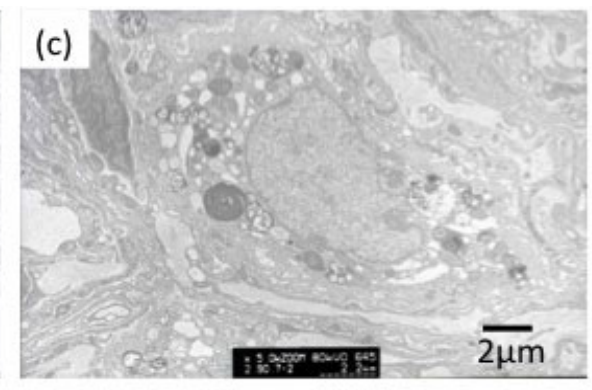

Figure 3. Alveolar macrophage in the rat lung after 1 week (a)-(b), and 3 months (c) instillation of SWCNTs. 